\title{
Asymmetric Double-Quantum-Well Phase Modulator Using Surface Acoustic Waves
}

\author{
Wallace C. H. Choy, E. Herbert Li, Senior Member, IEEE, and Bernard L. Weiss, Senior Member, IEEE
}

\begin{abstract}
An AlGaAs-GaAs asymmetric double-quantum-well (DQW) optical phase modulator using surface acoustic waves is investigated theoretically. The optimization steps of the DQW structure, which so far have not been reported in detail, are discussed here. The optimized phase modulator structure is found to contain a five-period QDW active region. A surface acoustic wave induces a potential field which provides the phase modulation. Analysis of the modulation characteristics show that by using the asymmetric DQW, the large change of the induced potential at the surface and thus large modification of the quantum-well $(\mathrm{QW})$ structure can be utilized. The modification of each QW structure is consistent, although this consistency is not always preserved in typical surface acoustic wave devices. Consequently, the change of refractive index in each of the five DQW's is almost identical. Besides, the change of effective refractive index is ten times larger here in comparison to a modulator with a five-period single $\mathrm{QW}$ as the active region and thus produces a larger phase modulation. In addition, a long wavelength and a low surface acoustic wave power required here simplify the fabrication of surface acoustic wave transducer and the acoustooptic phase modulator.
\end{abstract}

Index Terms-Acoustooptic device, double quantum well, electrooptical modulation, piezoelectric effect of surface acoustic waves, surface acoustic wave modulator.

\section{INTRODUCTION}

A COUSTOOPTIC devices, such as modulators, beam deflectors, and correlators, based on the interaction with surface acoustic waves (SAW's), have been developed for signal processing applications [1], and SAW technology provides the advantage of optoelectronic integration [2]. Recently, electrooptic modulators using the interaction of SAW's with III-V semiconductor multiple-quantum-well (MQW) structures have been demonstrated using the quantum-confined Stark effect [2], [3].

As we have reported [3], SAW induces both a potential and an electric field in the quantum-well $(\mathrm{QW})$ device structure which reduce nonuniformly with depth. The induced potential modifies the MQW structure and thus its optical properties. A more uniform change of each QW in the MQW structure can be obtained by increasing the SAW wavelength $\left(\lambda_{\mathrm{SAW}}\right)$.

Manuscript received Jan. 28, 1998; revised June 8, 1998. This work was supported by the Hong Kong RGC earmarked grant. The work of W. C. H. Choy was supported by a research scholarship from the Croucher Foundation.

W. C. H. Choy and B. L. Weiss are with the School of Electronic Engineering, Information Technology and Mathematics, University of Surrey, Guildford, Surrey, GU2 5XH, U.K.

E. H. Li is currently with the Division of Engineering and Applied Sciences, Harvard University, Cambridge, MA 02138 USA, on leave from the Department of Electrical and Electronic Engineering, University of Hong Kong, Pokfulam Road, Hong Kong.

Publisher Item Identifier S 0018-9197(98)07185-1.
However, the change of the QW properties with the induced potential reduce when $\lambda_{\mathrm{SAW}}$ increases, i.e., there is a tradeoff between the uniformity and the strength of the variation of the QW properties. In order to make use of the large change of the SAW induced potential at the device surface to produce large change of QW properties and to take account of the nonuniform nature of these QW variations, an asymmetric double-quantum-well (DQW) structure is proposed here for the SAW optical phase modulator. In addition, with the optimized structure using a DQW as the active region of the modulator, the optimum operation of the modulator only requires a long $\lambda_{\text {SAW }}(15 \mu \mathrm{m})$ and low SAW power $\left(3 \mathrm{~mW}\right.$ per $\left.\lambda_{\text {SAW }}\right)$ which greatly simplifies the fabrication of the device.

The asymmetric AlGaAs-GaAs DQW structure contains two QW's with different Al fractions separated by a thin inner barrier $(<50 \AA)$, and this type of structure has received considerable interest, both experimentally and theoretically [4], [5]. The coupling effects between the two wells of the asymmetric DQW provide a large refractive index change and thus a high phase modulation [4]. With an appropriate DQW structure, only a weak applied electric field $(\sim 20 \mathrm{kV} / \mathrm{cm})$ is required to produce a large refractive index change [5]. Therefore, the structure can be developed as the active region of a SAW modulator and a long $\lambda_{\mathrm{SAW}}$, and a low-power SAW can be used to generate the uniform electric field for useful optical modulators. However, the optimization of the asymmetric DQW structure for the low-power SAW based electrooptic modulators have not been discussed previously.

In this paper, the optimization of the asymmetric DQW are addressed by modeling its optical properties, including the absorption coefficient and refractive index for a range of QW parameters. It is proposed that the optimized asymmetric DQW acts as the active region of a waveguide-type SAW phase modulator and the modulation properties of this modulator are calculated. The DQW active region is designed at the surface of the modulator so that, apart from the advantage of requiring the long $\lambda_{\mathrm{SAW}}$ and the low power of SAW mentioned previously, a strong electric field can be obtained to produce the phase modulation.

\section{MODELING}

The QW structure consists of five periods of 105- $\AA$ DQW's each separated by $100-\AA \mathrm{Al}_{0.52} \mathrm{Ga}_{0.48} \mathrm{As}$ isolation barriers which is the active region of the waveguide-type SAW phase modulator, and this region is separated from the GaAs substrate by a thick $\mathrm{Al}_{0.52} \mathrm{Ga}_{0.48} \mathrm{As}$ lower cladding layer. A 200 - $\AA$-thick $\mathrm{Al}_{0.52} \mathrm{Ga}_{0.48} \mathrm{As}$ layer is finally grown on the 
surface of the structure. The SAW interdigital transducer is deposited at one side of the device structure to generate the SAW's. The growth direction of the DQW's is $\langle 100\rangle$ and the SAW propagates along the $\langle 110\rangle$ direction. The incident light propagates normal to the propagation direction of the SAW. It should be noted that the $\mathrm{ZnO}$ thin layer depositing on the top surface of the device structure can be used to enhance the SAW-induced potential and thus the modulation strength [3], [6], although it is out of the scope here to study its effects in the device structure.

The perturbation of the DQW subband structure by SAW is modeled so that electroabsorption and electrooptic variations due to SAW induced piezoelectric and elastooptic effects on the DQW can be determined. The modulation of the optical wave depends on the interaction of the optical field with the SAW perturbed DQW structure, and therefore the one-dimensional Maxwell's equation is solved analytically. Consequently, the optical guided mode, the change of effective refractive index, and the change of the effective absorption coefficient are obtained for the determination of the modulation characteristics of the phase modulator.

\section{A. SAW-Perturbed DQW Structure}

The propagation of SAW is described by the equation of motion of particles in elastic medium and the electric displacement equation in a medium [7], [8]. For the subband structure of a DQW, it is considered that the isolation layer separating two DQW structures is so thick that there is no significant coupling between adjacent DQW's, and therefore a DQW model can be used here. The QW subband edge states in the $\Gamma$-valley are calculated by solving a one-dimensional (1D) one-particle Schrödinger-like equation numerically. This equation is reexpressed as a tridiagonal matrix using the Strum-Liouville method [9], and eigenvalues of the tridiagonal matrix are found by using the recursive method [10] followed by a bisection method. An inversion iteration [10] is then used to improve the eigenvalue accuracy and find the eigenvector, i.e., the envelope function of an electron. The SAW induces both strain and an electric field in the AlGaAs-GaAs piezoelectric heterostructure. However, the induced strains are too small $(<0.1 \%)$ to significantly modify the subband structure heterostructure and to change the semiconductor material bandgap [11]. Consequently, only the potential due to the SAW-induced electric field is considered as an additional linear perturbation term in modifying the DQW potential profile.

The exciton in a DQW is modeled using the variational method which has been discussed previously [12]. When the energy difference between the first and second subband states is small, the intersubband coupling of the excitons must be considered. Consequently, the variational wavefunction associated with the lowest two subband states of the electron and the hole are chosen as [13]

$$
\psi_{C_{i} V_{j}}(\rho)=\sum_{C_{i}=1}^{N} \sum_{V_{j}=1}^{M} d_{i} \chi_{C_{i}}\left(z_{e}\right) f_{j} \chi_{V_{j}}\left(z_{h}\right) e^{-\beta \rho}
$$

where $\rho$ is the displacement of the position vectors of an electron and a hole projected in the $x-y$ plane, $N, M$ are the number of electron states and hole states in calculation and both have a value of two, $\chi_{C_{i}}\left(z_{e}\right)$ and $\chi_{V_{j}}\left(z_{h}\right)$ are the wavefunctions of the $C_{i}$ th conduction subband and $V_{j}$ th valence subband for heavy and light holes, respectively, $\beta$ is a variational parameter governing the radial extent of the wavefunction, and $d_{i}$ and $f_{j}$ are variational parameters which are subject to the normalization constraints

$$
\sum_{i=1}^{N}\left|d_{i}\right|^{2}=1 \quad \text { and } \quad \sum_{j=1}^{N}\left|f_{j}\right|^{2}=1 .
$$

The minimized expectation value of the exciton Hamiltonian $\zeta$ which is the total exciton energy can be determined by

$$
\zeta=\frac{\left\langle\psi\left|H_{e x c}\right| \psi\right\rangle}{\langle\psi \mid \psi\rangle}
$$

The exciton binding energy $E_{b}$ is then obtained

$$
E_{b}=E_{1}^{e}+E_{1}^{h}-\zeta
$$

where $E_{1}^{e}$ and $E_{1}^{h}$ are the first subband energy of an electron and a hole, respectively.

\section{B. Optical Properties}

The subband structure and the exciton states, including intersubband coupling effects, are used to calculate the absorption coefficient $(\alpha)$, the unbiased absolute refractive index $\left(n_{r}\right)$, the change of absorption coefficient $(\Delta \alpha)$, and the change of refractive index $(\Delta n)$ due to an electric field for the DQW structure. Details of $\alpha$ and $n_{r}$ calculations are in [14] and [15], respectively. The essential steps for $\alpha$ and $n_{r}$ of the DQW are presented here. The bound state $\alpha$ can be obtained from the imaginary part of the dielectric constant $\varepsilon_{2}^{\text {bound }}(\omega)$ [16], where the superscript denotes the conduction-valance band bound state

$$
\begin{aligned}
\varepsilon_{2}^{\text {bound }}(\omega)= & \frac{e^{2} \mu_{ \pm}}{6 \varepsilon_{o} m_{e}^{*} E_{C V}^{2}\left(L_{w 1}+L_{w 2}+L_{i b}\right)} \\
& \cdot\left(\frac{E_{g}\left(E_{g}+\Delta_{0}\right)}{E_{g}+\frac{2}{3} \Delta_{0}}\right) \sum_{C, V}\left|\left\langle\chi_{C} \mid \chi_{V}\right\rangle\right|^{2} I_{C V}(\hbar \omega)
\end{aligned}
$$

where

$$
\begin{aligned}
I_{C V}(\hbar \omega) & =\int_{0}^{\infty} \wp(E) S(E) L(E) d E, \\
L(E) & =\frac{\Gamma_{B}}{\pi\left\{\left[E_{C V}+E-\hbar \omega\right)^{2}+\Gamma_{B}^{2}\right\}}
\end{aligned}
$$

and $L_{w 1}, L_{w 2}$ and $L_{i b}$ are the thickness of the first and second wells, and inner barrier, respectively, $\left|\left\langle\chi_{C} \mid \chi_{V}\right\rangle\right|^{2}$ is the probability of the wavefunction overlap between $\chi_{C}$ and $\chi_{V}, S(E)$ is the Sommerfield enhancement factor which is assumed here to be a constant, $L(E)$ is the Lorentzian broadening factor, $\Gamma_{B}$ is the bound state linewidth (half width half maximum, HFHM) broadening factor, $\wp(E)$ is the polarization factor, $\varepsilon_{O}$ is the permittivity of free space, $E_{C V}$ is the transition energy between a conduction subband and a valence subband, and $\Delta_{o}, m_{e}^{*}$, and $E_{g}$ are the spin-orbit splitting gap, the effective electron mass, and bandgap energy, 
respectively. The latter three parameters are calculated by weighing the ratio between the well widths and the $\mathrm{Al}$ fraction of the two wells. Details of the polarization factor can be found in [14]. The imaginary part of the DQW exciton dielectric constant $\varepsilon_{2}^{\text {exc }}(\omega)$, implied the exciton absorption coefficient, is given by

$$
\begin{aligned}
\varepsilon_{2}^{\operatorname{exc}}(\omega)= & \frac{e^{2} \hbar^{2}}{2 \varepsilon_{o} m_{e}^{*} E_{C V}^{2}\left(L_{w 1}+L_{w 2}+L_{i b}\right)} \\
& \cdot\left(\frac{E_{g}\left(E_{g}+\Delta_{o}\right)}{E_{g}+\frac{2}{3} \Delta_{o}}\right) \frac{\frac{2 \beta^{2}}{\pi}\left|\psi_{C_{i} V_{j}}(\rho)\right|^{2}{ }_{\wp} \Gamma_{X B}}{\pi\left[\left(E_{\mathrm{exc}}-\hbar \omega\right)^{2}+\Gamma_{X B}^{2}\right]}
\end{aligned}
$$

where $E_{\text {exc }}$ is the excitonic transition energy and $\Gamma_{X B}$ is the exciton linewidth (HWHM) broadening factor.

The dielectric functions are used to determine $n_{r}$ [15], [17], which is given by

$$
n_{r}(\omega)=\left(\frac{1}{2} \varepsilon_{1}(\omega)+\frac{1}{2}\left\{\left[\varepsilon_{1}(\omega)\right]^{2}+\left[\varepsilon_{2}(\omega)\right]^{2}\right\}^{1 / 2}\right)^{1 / 2}
$$

where $\varepsilon_{1}$ and $\varepsilon_{2}$ are the real and imaginary parts of the dielectric function respectively, and are given by

$$
\begin{aligned}
& \varepsilon_{1}(\omega)=\varepsilon_{1}^{\Gamma}(\omega)+\varepsilon_{1}^{L}(\omega)+\varepsilon_{1}^{X}(\omega)+\varepsilon_{\infty} \\
& \varepsilon_{2}(\omega)=\varepsilon_{2}^{\Gamma}(\omega)+\varepsilon_{2}^{L}(\omega)+\varepsilon_{2}^{X}(\omega)+\varepsilon_{2}^{I D}
\end{aligned}
$$

where $\varepsilon_{1}^{\Gamma}$ and $\varepsilon_{2}^{\Gamma}$ denotes the contribution from $\Gamma$-valley, $\varepsilon_{1}^{L}$ and $\varepsilon_{2}^{L}$ denotes the contribution from $L$-valley, $\varepsilon_{1}^{X}$ and $\varepsilon_{2}^{X}$ denotes the contribution from $X$-valley, $\varepsilon_{\infty}$ denotes the contribution from other transitions to $\varepsilon_{1}$, and $\varepsilon_{2}^{I D}$ denotes the contribution from the indirect transitions to $\varepsilon_{2}$. The quantum confinement affects primarily the contribution of the transitions in the $\Gamma$-valley, and thus $\varepsilon_{1}^{\Gamma}$ and $\varepsilon_{2}^{\Gamma}$, while the contributions of the bulk-like $L$ - and $X$-valleys and all other transitions above the DQW, which contribute to the real and imaginary dielectric functions, are obtained using a bulk calculation [18].

By considering the effects contributed by the DQW well layers and the bulk barrier layers, the imaginary part of the dielectric constant contributed by the $\Gamma$-valley is given by

$$
\varepsilon_{2}^{\Gamma}(\omega)=\varepsilon_{2}^{\mathrm{exc}}(\omega)+\varepsilon_{2}^{\mathrm{bound}}(\omega)+\varepsilon_{2}^{\Gamma \text { bulk }}(\omega)
$$

where the superscript $\Gamma_{\text {bulk }}$ refers to the contribution due to the bulk-like higher energy region above the QW. The expressions for $\varepsilon_{2}^{\text {exc }}$ and $\varepsilon_{2}^{\text {bound }}$ are given by (5) and (6), respectively, and

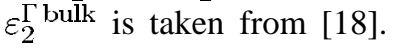

In the modeling of the DQW, the $\varepsilon_{2}^{\Gamma \text { bulk }}(\omega)$ is determined by weighing the barrier and well together in the ratio of the width of the two wells of a DQW and width of barriers

$$
\begin{aligned}
\varepsilon_{2}^{\Gamma \text { bulk }}(\omega)= & \frac{L_{w 1}}{L_{\text {tot }}}\left[\varepsilon_{2}^{\Gamma \text { bulk }}(\omega)\right]_{w 1}+\frac{L_{w 2}}{L_{\text {tot }}}\left[\varepsilon_{2}^{\Gamma \text { bulk }}(\omega)\right]_{w 2} \\
& +\frac{L_{i b}}{L_{\text {tot }}}\left[\varepsilon_{2}^{\Gamma \text { bulk }}(\omega)\right]_{i b}+\frac{2 L_{o b}}{L_{\text {tot }}}\left[\varepsilon_{2}^{\Gamma \text { bulk }}(\omega)\right]_{o b}
\end{aligned}
$$

where $L_{o b}$ is the thickness of one outer barrier and $L_{\text {tot }}=$ $L_{w 1}+L_{w 2}+2 L_{o b}+L_{i b}$, the subscripts $w 1, w 2, i b$, and $o b$ denote the $\varepsilon_{2}^{\Gamma \text { bulk }}(\omega)$ of first well, second well, inner barrier layer, and isolation layer respectively, and $\varepsilon_{1}^{\Gamma}(\omega)$ is determined using the Kramers-Krönig transformation of $\varepsilon_{2}^{\Gamma}(\omega)$; the real part of refractive index is evaluated from (7) and includes contributions of the real and imaginary dielectric functions by the $X$ - and $L$-valleys and higher order transitions obtained from the bulk calculation [18]. In order to verify the refractive index model, the calculated refractive index is compared with experimental values of a single QW taken from [19]. By setting $L_{i b}$ and $L_{w 2}=0$, the calculated $n_{r}$ demonstrates good agreement between the model and experiment [15]. SAW induced modifications of the absorption coefficient are determined using (5) and (6). The change of refractive index induced by SAW is then calculated by using the Kramers-Krönig transformation from the change of the absorption coefficient.

\section{Modulator Characteristics}

A multilayer planar waveguide model using the transfer matrix method [16] is used. From this calculation, the mode field profiles and the propagation constants are obtained for the determination of the effective absorption coefficient $\alpha_{\text {eff }}$ and effective refractive index $n_{\text {eff }}$, respectively [3], to enable the performance of the phase modulator to be calculated. The important performance characteristics include the phase change $\Delta \phi$, the optical confinement factor, the chirp parameter $\beta_{\text {mod }}$, and the absorption loss $\alpha_{\text {loss }}$. Details of these parameters can be found in [3].

A typical electrooptical phase modulator requires $\beta_{\bmod }>$ 10 [20]. High-performance phase modulators also require a low $\alpha_{\text {loss }}$, which is a measure of the insertion loss of the device, where $\alpha_{\text {loss }}$ is defined as $\alpha_{\text {eff }}$ with the SAW effect.

\section{RESULTS AND DisCUSSIONS}

A five-period asymmetric DQW phase modulator operated by using a SAW with $\lambda_{\text {SAW }}$ of $15 \mu \mathrm{m}$ and SAW power of $3 \mathrm{~mW}$ per $\lambda_{\text {SAW }}$ is analyzed here. In order to have a low $\alpha_{\text {loss }}$, the phase modulator is operated at a wavelength beyond the exciton absorption peak of the $\mathrm{C} 1-\mathrm{HH} 1$ transition [20]. The optimization of the DQW structure is discussed first and the modulation characteristics of the SAW modulator with the optimized DQW as its active region are then addressed. Since $\lambda_{\text {SAW }}$ is $15 \mu \mathrm{m}$ and a single DQW thickness is $\sim 10^{-2} \mu \mathrm{m}$, the SAW induced potential due to the piezoelectric effect is effectively linear in each DQW which are in the top $0.2 \mu \mathrm{m}$ of the structure. Therefore, DQW potential profile tilted by an uniform bias electric field, as in a conventional $\mathrm{p}-\mathrm{i}-\mathrm{n}$ reverse bias modulator [14], [16], is studied in optimization of the DQW. In the modeling of SAW, also due to the considerable difference of the $\lambda_{\text {SAW }}$ magnitude and DQW dimension, SAW is relatively insensitive to the presence of the thin inner barrier of the DQW. The SAW potential profile is therefore modeled using a five-period single QW structure where the well width is equivalent to the thickness of the DQW structure.

\section{A. Optimization of Asymmetric $D Q W$}

In order to improve the optical properties of a DQW structure, the structural parameters, including the well widths, the $\mathrm{Al}$ fraction in wells, the $\mathrm{Al}$ fraction of the isolation barriers 
(a)

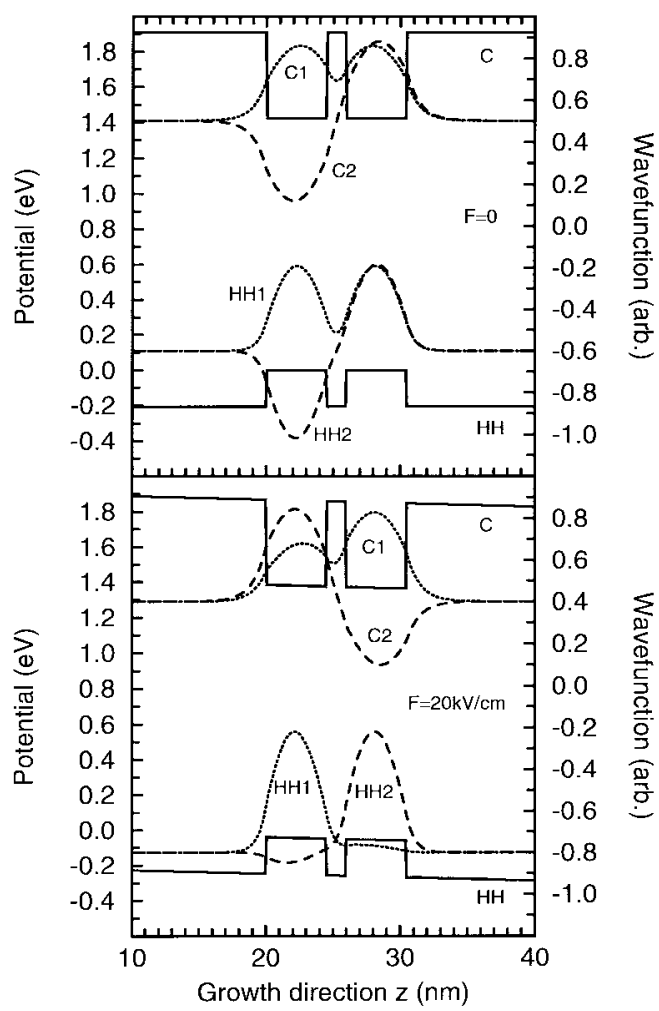

Fig. 1. Potential profile and wavefunctions of the symmetric structure A: Z45/15/45, X0.0/0.52/0.0 bounded by two outer barriers with Al fraction of 0.52 at field (a) $F=0$ and (b) $F=20 \mathrm{kV} / \mathrm{cm}$. Potential profiles (solid line), $\mathrm{C} 1$ and $\mathrm{HH} 1$ wavefunctions (dotted line), and $\mathrm{C} 2$ and $\mathrm{HH} 2$ wavefunctions (dashed line).

between the two adjacent DQW pairs, and the thickness and $\mathrm{Al}$ fraction of the inner barrier have to be optimized. Generally, the confinement of an envelope wavefunction improves when the $\mathrm{Al}$ fraction of the isolation barriers increases and the total thickness $\left(L_{w 1}+L_{w 2}+L_{i b}\right)$ reduces. The effects of varying the thickness of the wells and inner barrier of a symmetric DQW on the optical properties have been thoroughly studied [21] and are not discussed further here. In order to have a strong quantum confinement, an $\mathrm{Al}$ fraction of 0.52 is used for the isolation barriers [5] and the thickness of $\left(L_{w 1}+L_{w 2}+L_{i b}\right)$ is $105 \AA(\sim 100 \AA)$ [21]. The inner barrier thickness of 15 $\AA$ is used here to produce strong coupling effects in the DQW structure [21]. In order to optimize an asymmetric DQW structure, the effects of the two well widths $L_{w 1}$ and $L_{w 2}$, the $\mathrm{Al}$ fraction of the two wells, and the inner barrier are investigated here. An optimized DQW structure is obtained by analyzing the wavefunction distribution, the square of the overlap integral, the square of variational parameter $\beta^{2}$ (which governs the radial extent of the wavefunction), and the absorption coefficient.

Four AlGaAs-GaAs DQW structures are used to show the effects of the $\mathrm{Al}$ fraction of the well and barrier layers and the well width of DQW on its optical properties. They are: A) two identical GaAs wells (Al fraction ) with widths of $45 \AA$, an inner barrier width of $15 \AA$, and an Al fraction of 0.52 ; this structure is denoted as $\mathrm{Z} 45 / 15 / 45$ and $\mathrm{X} 0.0 / 0.52 / 0.0$ for layer dimension and $\mathrm{Al}$ fraction respectively; b) Z30/15/60 and $\mathrm{X} 0.0 / 0.52 / 0.0$ to show the effect of different well widths; c)
TABLE I

Transition Energies $E_{\text {tr }}$ AND SQuare of the OverlapPing INTEGRAL, OVINT, OF FOUR DQW'S STRUCTURES-A: Z45/15/45, X0.0/0.52/0.0; B: Z30/15/60, X0.0/0.52/0.0; C: Z30/15/60, X0.0/0.52/0.036; D: Z30/15/60, X0.0/1.0/0.036

\begin{tabular}{ll|llll|llll}
\hline \multirow{2}{*}{ Cases } & $\mathrm{F}=0$ & & & & \multicolumn{2}{|l}{$\mathrm{F}=20 \mathrm{kV} / \mathrm{cm}$} & & \\
& & $\mathrm{C} 1-$ & $\mathrm{C} 1-$ & $\mathrm{C} 2-$ & $\mathrm{C} 2$ & $\mathrm{C} 1-$ & $\mathrm{C} 1-$ & $\mathrm{C} 2-$ & $\mathrm{C} 2-$ \\
& $\mathrm{HH1}$ & $\mathrm{HH} 2$ & $\mathrm{HH1}$ & $\mathrm{HH} 2$ & $\mathrm{HH1}$ & $\mathrm{HH} 2$ & $\mathrm{HH} 1$ & $\mathrm{HH2}$ \\
\hline $\mathrm{A}$ & $\mathrm{E}_{\mathrm{tI}}(\mathrm{eV})$ & 1.538 & 1.540 & 1.566 & 1.568 & 1.532 & 1.544 & 1.562 & 1.574 \\
& OVINT & 0.963 & 0.000 & 0.000 & 0.975 & 0.345 & 0.618 & 0.622 & 0.350 \\
$\mathrm{~B}$ & $\mathrm{E}_{\mathrm{t}}(\mathrm{eV})$ & 1.512 & 1.538 & 1.610 & 1.636 & 1.512 & 1.526 & 1.620 & 1.635 \\
& OVINT & 0.962 & 0.013 & 0.015 & 0.937 & 0.967 & 0.004 & 0.003 & 0.930 \\
$\mathrm{C}$ & $\mathrm{E}_{\mathrm{tr}}(\mathrm{eV})$ & 1.567 & 1.575 & 1.630 & 1.639 & 1.564 & 1.568 & 1.637 & 1.641 \\
& OVINT & 0.957 & 0.018 & 0.020 & 0.930 & 0.163 & 0.808 & 0.780 & 0.156 \\
$\mathrm{D}$ & $\mathrm{E}_{\mathrm{tI}}(\mathrm{eV})$ & 1.661 & 1.687 & 1.670 & 1.695 & 1.655 & 1.693 & 1.664 & 1.702 \\
& OVINT & 0.834 & 0.126 & 0.124 & 0.854 & 0.112 & 0.856 & 0.847 & 0.113 \\
\hline
\end{tabular}

$\mathrm{Z} 30 / 15 / 60$ and $\mathrm{X} 0.0 / 0.52 / 0.036$ to show the effects of different well composition; and D) Z30/15/60 and X0.0/1.0/0.118 to show the effect of the composition of the barrier layer. The isolation layers of all four DQW structures have an $\mathrm{Al}$ fraction of 0.52 .

The potential profile of structure A, Z45/15/45 and $\mathrm{X} 0.0 / 0.52 / 0.0$, is shown in Fig. 1. The square of the overlap integral of the diagonal transitions, i.e., $\mathrm{C} 1-\mathrm{HH} 1$ and $\mathrm{C} 2-\mathrm{HH} 2$, reduce and that of the nondiagonal transitions, i.e., $\mathrm{C} 1-\mathrm{HH} 2$ and $\mathrm{C} 2-\mathrm{HH} 1$, increase when the uniform electric field $F$ increases from 0 to $20 \mathrm{kV} / \mathrm{cm}$, as shown in Table I. These changes can be expressed by plotting their wavefunctions, see Fig. 1. Although the square of the overlap integral of the LH transitions has not been discussed here, the variations are similar to those of the HH transitions. The exciton absorption spectra of structure A applied fields of $F=0$ and $20 \mathrm{kV} / \mathrm{cm}$ are shown in Fig. 2(a). The exciton absorption coefficient $\alpha_{\text {exc }}$ of the diagonal transitions, such as $\mathrm{C} 1-\mathrm{HH} 1$, reduce considerably and $\alpha_{\text {exc }}$ for the nondiagonal transitions, such as C1-HH2, establishes with a high magnitude when $F$ increases to $20 \mathrm{kV} / \mathrm{cm}$. The change of refractive index due to the uniform applied electric field is obtained and shown in Fig. 3(a).

When the widths of the two wells of the symmetric structure A are modified to become asymmetric, as in structure B, $\mathrm{Z} 30 / 15 / 60$ and $\mathrm{X} 0.0 / 0.52 / 0.0$, the square of the overlap integral of the diagonal and nondiagonal transitions show no significant change due to the uniform applied field, as shown in Table I. When the applied electric field in structure A (symmetric DQW) increases from 0 to $20 \mathrm{kV} / \mathrm{cm}$, the square of the overlap integral of the diagonal transitions reduces from $\sim 0.9$ to $\sim 0.6$ and the nondiagonal ones increase from 0 to $\sim 0.6$. However, by modifying the symmetric DQW to the asymmetric DQW structure B, the square of the overlap integral is maintained at $\sim 0.9$ and $\sim 0$ for diagonal and nondiagonal transitions, respectively, when the applied field increases from 0 to $20 \mathrm{kV} / \mathrm{cm}$. As a result, $\alpha_{\text {exc }}$ spectra with and without the applied field are similar to each other and result in a small refractive index change for structure B, as shown in Fig. 3(b). Consequently, an asymmetric DQW produced by only adjusting the well widths is not useful for phase modulation due to the small SAW induced field $(\sim 20 \mathrm{kV} / \mathrm{cm})$.

By increasing the $\mathrm{Al}$ fraction in the wider well to 0.036, i.e., structure $\mathrm{C}$ with $\mathrm{Z} 30 / 15 / 60$ and $\mathrm{X} 0.0 / 0.52 / 0.036$, the change of the square of its overlap integral due to the applied field is 


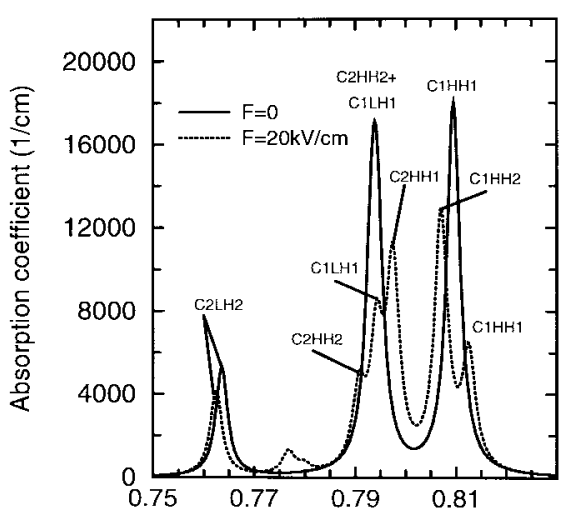

(a)

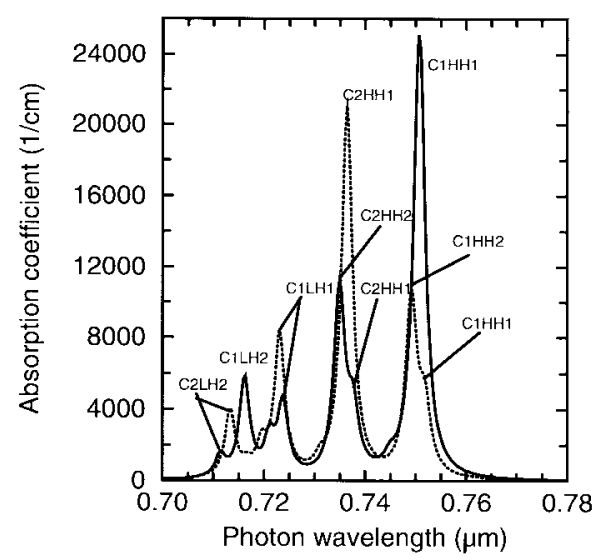

(b)

Fig. 2. Exciton absorption spectra of structures: (a) A at $F=0$ (solid line) and $F=20 \mathrm{kV} / \mathrm{cm}$ (dotted line) and (b) $\mathrm{D}$ at $F=0$ (solid line) and $F=20 \mathrm{kV} / \mathrm{cm}$ (dotted line).

(a)

(b)

(c)

(d)

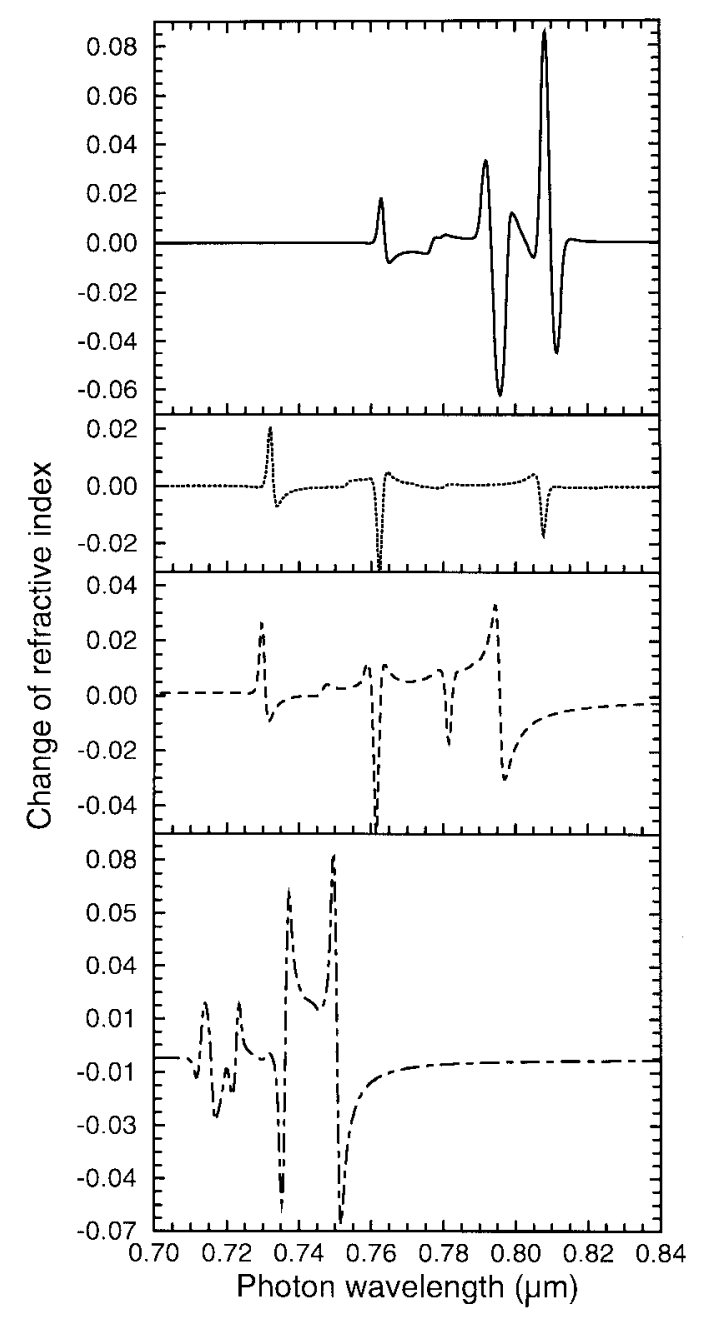

Fig. 3. Change of refractive index spectra of the four DQW structures (a) A (solid line), (b) B (dotted line), (c) C (dashed line), and (d) D (dotted-dashed line).

similar in trend to that of structure A. However, in structure $\mathrm{C}$, the total change of the square of the overlap integral for structure $\mathrm{C}$ is larger than that for structure A. Therefore, by increasing the $\mathrm{Al}$ fraction of the wider well to 0.036, the change of the square of the overlap integral due to an applied electric field on both the diagonal and the nondiagonal transitions can increase to $\sim 0.8$. The field-induced changes in symmetric structure A are only $\sim 0.6$ for both diagonal and nondiagonal transitions. If the $\mathrm{Al}$ fraction of the wider well increases further, the change of the square of the overlap integral reduces again, just as for the case when the $\mathrm{Al}$ fraction increases to 0.12 , the change of the square of the overlap integral of $\mathrm{C} 1-\mathrm{HH} 1$ and $\mathrm{C} 1-\mathrm{LH} 1$ are from $\sim 0.62$ to $\sim 0.41$ and from $\sim 0.32$ to $\sim 0.53$, respectively. Therefore, the change of the absorption coefficient and refractive index are reduced. Consequently, the large change of the square of the overlap integral contributes to a large refractive index change for structure $\mathrm{C}$ at wavelengths $>0.795 \mu \mathrm{m}$ [see Fig. 3(c)], as compared to that of structure A at wavelengths $>0.815 \mu \mathrm{m}$ [see Fig. 3(a)]. Thus structure $\mathrm{C}$ is more useful for developing a phase modulator.

It is interesting to note that a larger refractive index change can be obtained using the optimized DQW structure D, $\mathrm{Z} 30 / 15 / 60$ and $\mathrm{X} 0.0 / 1.0 / 0.118$, where the most important difference between structures $\mathrm{C}$ and $\mathrm{D}$ is the increase of the Al fraction of the inner barrier to 1.0. As shown in Fig. 4(a), the $\mathrm{C} 1$ and $\mathrm{HH} 1$ wavefunctions for $F=0$ are predominantly in the narrow well because of increases of the $\mathrm{Al}$ fraction of both the barrier and the wider well, while in structure A the two wavefunctions are evenly distributed in both wells, as shown in Fig. 1(a). For $F=20 \mathrm{kV} / \mathrm{cm}$ [see Fig. 4(b)], $\mathrm{C} 1$ of structure $\mathrm{D}$ is better confined in the right-hand well as compared to that of structure A [see Fig. 1(a)], since the Al fraction of the inner barrier and width of the right-hand well of structure D increases and broadens respectively, while HH1 has no significant change. Consequently, when the $\mathrm{Al}$ fraction of the inner barrier increases to 1.0 (structure D), the square of the overlap integral of $\mathrm{C} 1-\mathrm{HH} 1$ reduces slightly to $\sim 0.83$ as compared to $\sim 0.96$ for the other structures for zero applied field (see Table I). However, since $\beta^{2}$ of C1-HH1 increases from $4.73 \times 10^{-5}$ in structure $A$ to $8.76 \times 10^{-5}$ in structure D, i.e., almost a two-fold increase of $\beta^{2}$, the C1-HH1 $\alpha_{\text {exc }}$ of structure $\mathrm{D}$ increases considerably to $\sim 25000 \mathrm{~cm}^{-1}$, as shown in Fig. 2(b). When $F=20 \mathrm{kV} / \mathrm{cm}$, the square of the overlap integral of $\mathrm{C} 1-\mathrm{HH} 1$ of structure D is one-third that of 
(a)

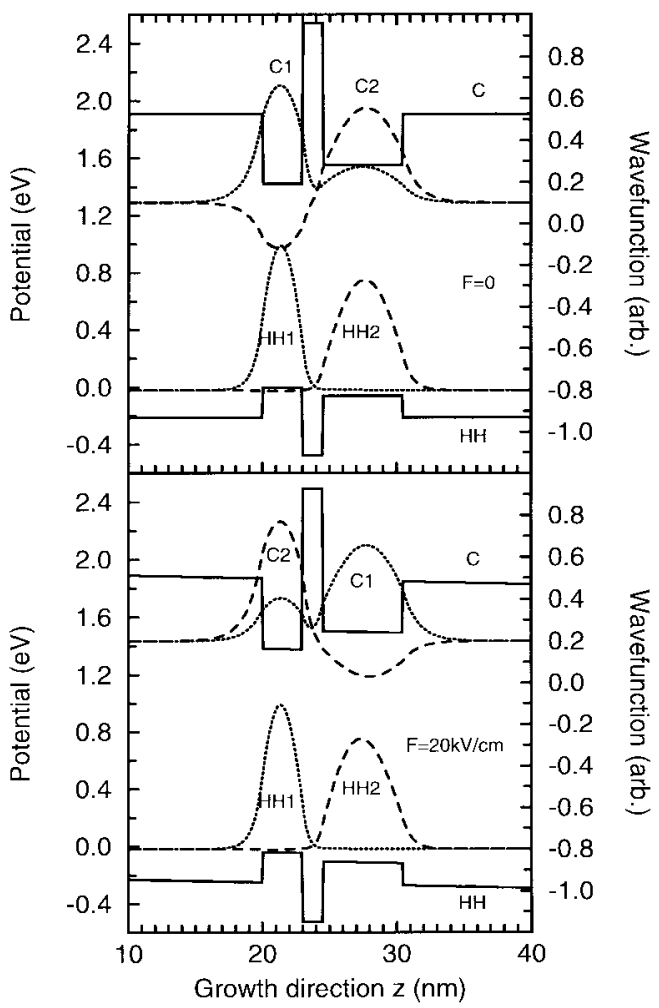

Fig. 4. Potential profile and wavefunctions of the optimized asymmetric structure D: Z30/15/60, X0.0/1.0/0.118 bounded by two outer barriers with an Al fraction of 0.52 at field (a) $F=0$ and (b) $F=20 \mathrm{kV} / \mathrm{cm}$. Potential profiles (solid line), $\mathrm{C} 1$ and $\mathrm{HH} 1$ wavefunctions (dotted line), and $\mathrm{C} 2$ and $\mathrm{HH} 2$ wavefunctions (dashed line).

structure $\mathrm{A}$ and $\beta^{2}$ of $\mathrm{C} 1-\mathrm{HH} 1$ is also slightly reduced from $3.99 \times 10^{-5}$ in structure A to $3.76 \times 10^{-5}$ in structure $\mathrm{D}$. The $\mathrm{C} 1-\mathrm{HH} 1 \alpha_{\mathrm{exc}}$ of structure D therefore reduces significantly, as shown in Fig. 2(b). It should be noted that since increasing the $\mathrm{Al}$ fraction in the inner barrier contributes to the variations of $\mathrm{C} 1$ and $\mathrm{HH} 1$ of both $F=0$ and $F=20 \mathrm{kV} / \mathrm{cm}$, structure $\mathrm{D}$ can be considered as a DQW to study the effect of the $\mathrm{Al}$ fraction in the barrier in optimizing the DQW structure, while the effects of increasing the well width and the $\mathrm{Al}$ fraction of the right-hand well have been discussed in structures B and $\mathrm{C}$, respectively.

The increase and decrease of the $\mathrm{C} 1-\mathrm{HH} 1 \alpha_{\text {exc }}$ of structure D for $F=0$ and $F=20 \mathrm{kV} / \mathrm{cm}$, respectively, result in a larger refractive index change at wavelengths between $0.75 \mu \mathrm{m}$ and $0.78 \mu \mathrm{m}$ for structure D as compared to that of structure A at wavelengths between 0.815 and $0.84 \mu \mathrm{m}$. The main reasons are the following. First, the large negative $\Delta \alpha_{\mathrm{exc}}$, i.e., $\alpha_{\mathrm{exc}}(F=$ $20 \mathrm{kV} / \mathrm{cm})-\alpha_{\text {exc }}(F=0)$, at wavelengths between $0.75 \mu \mathrm{m}$ and $0.76 \mu \mathrm{m}$ for structure D [see Fig. 2(b)] contributes to a large negative refractive index change at wavelengths between $0.75 \mu \mathrm{m}$ and $0.78 \mu \mathrm{m}$ after the Kramer-Krönig transformation, see Fig. 3(d). Second, the large but opposite sign $\Delta \alpha_{\text {exc }}$ of structure A for wavelengths between $0.80 \mu \mathrm{m}$ and 0.82 $\mu \mathrm{m}$ [see Fig. 2(a)] causes a cancellation of refractive index changes in the Kramer-Krönig transformation and results in a small positive refractive index change at wavelengths between $0.815 \mu \mathrm{m}$ and $0.84 \mu \mathrm{m}$ [see Fig. 3(a)]. As a consequence, structure $\mathrm{D}$ provides a larger refractive index for a low applied-

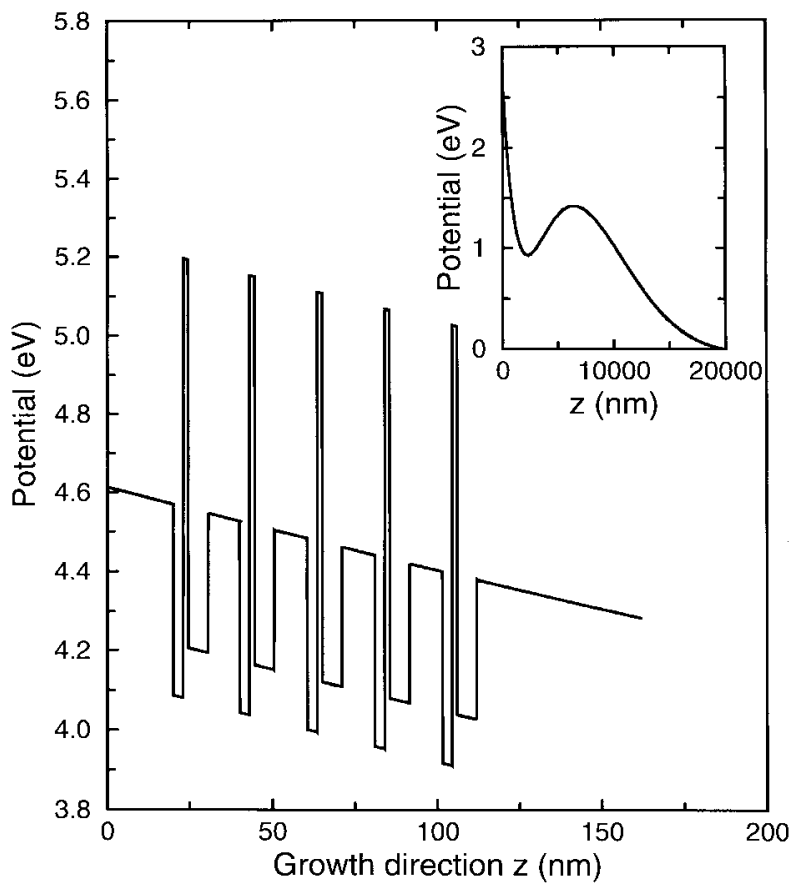

Fig. 5. Potential profile of the five-period DQW structures due to the SAW induced potential for $\lambda_{\mathrm{SAW}}$ of $15 \mu \mathrm{m}$ and SAW power of $3 \mathrm{~W}$. The inserted figure is the potential induced by the SAW.

field phase modulation than structure A. It should be noted that the expression for the large refractive index change of structure $\mathrm{D}$ is applicable to structure $\mathrm{C}$.

\section{B. Modulator Characteristics}

The optimized DQW structure contains five DQW periods as the active region of the SAW modulator. The five DQW's are tilted by the potential of the SAW induced electric field and they are located at the top region (with thickness $<200$ $\mathrm{nm}$ ) of the whole nonlinear SAW potential profile, as shown in Fig. 5. In this figure, the slope of the SAW potential is steepest at the surface, i.e., the strongest electric field induced by the SAW can used for phase modulation.

As shown in Fig. 6, $\alpha$ of the DQW's are modified due to the SAW induced potential. The absorption spectra of the first, third, and fifth DQW almost coincide, i.e., the slope of the SAW induced potential is almost identical over the five DQW's. This implies that each of the five DQW's can provide similar $\Delta n$, as shown in Fig. 7.

Although the optical confinement factor of the SAW phase modulator is not especially large $(\sim 0.54)$, the strong electric field induced by the SAW provides a large phase modulation, as shown in Table II. At an operating wavelength $\left(\lambda_{\mathrm{op}}\right)$ of $0.755 \mu \mathrm{m}, \Delta n_{\mathrm{eff}}$ and thus $\Delta \phi$ for a SAW aperture (modulation length) of $50 \mu \mathrm{m}$ are very high with values of $1.38 \times 10^{-2}$ and $5.76 \mathrm{rad}$, respectively. However, the penalty is a large $\Delta \alpha_{\text {eff }}$ with a value of $329 \mathrm{~cm}^{-1}$ which results in a low $\beta_{\text {mod }}=$ $6.9(<10)$. This means that, although a large phase modulation is produced, an unexpected strong intensity modulation is simultaneously produced. Therefore, it is not useful for a SAW phase modulator to operate at $\lambda_{\mathrm{op}}=0.755 \mu \mathrm{m}$. For longer $\lambda_{\mathrm{op}}, \beta_{\mathrm{mod}}$ increases which implies that the phase modulation 


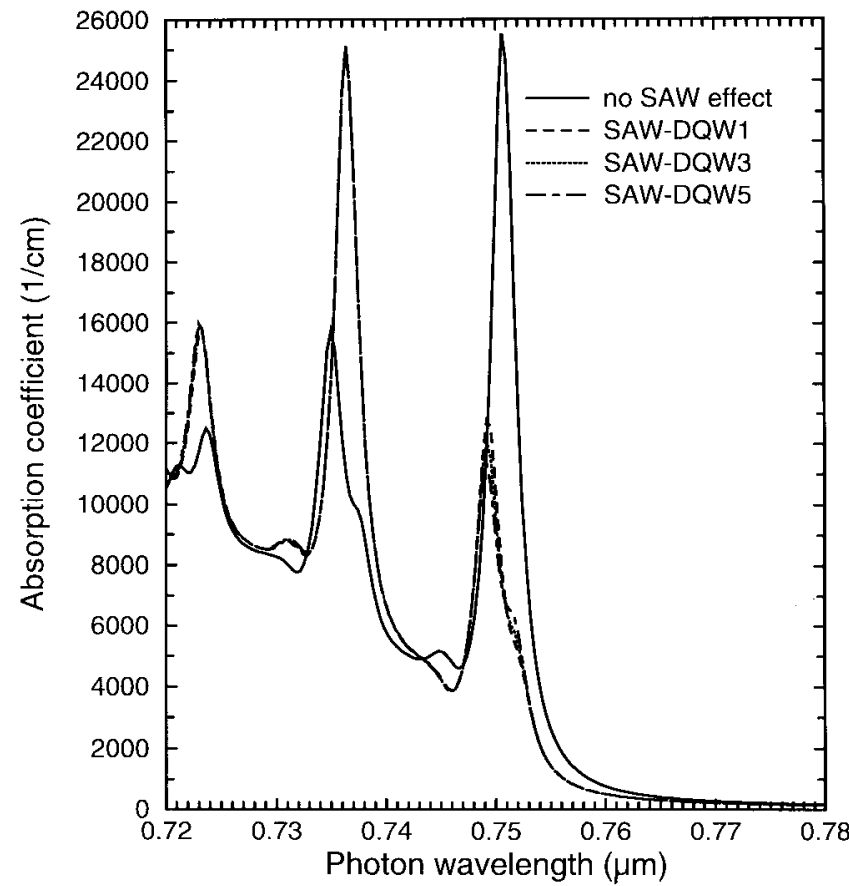

Fig. 6. The absorption coefficient $\alpha$ spectra of the five DQW's in the active region of the SAW phase modulator. $\alpha$ of the DQW without SAW effect (solid line), $\alpha$ of the first DQW with the SAW effect (dashed line), $\alpha$ of the third DQW with the SAW effect (dot line), and $\alpha$ of the fifth DQW with the SAW effect (dotted-dashed line).

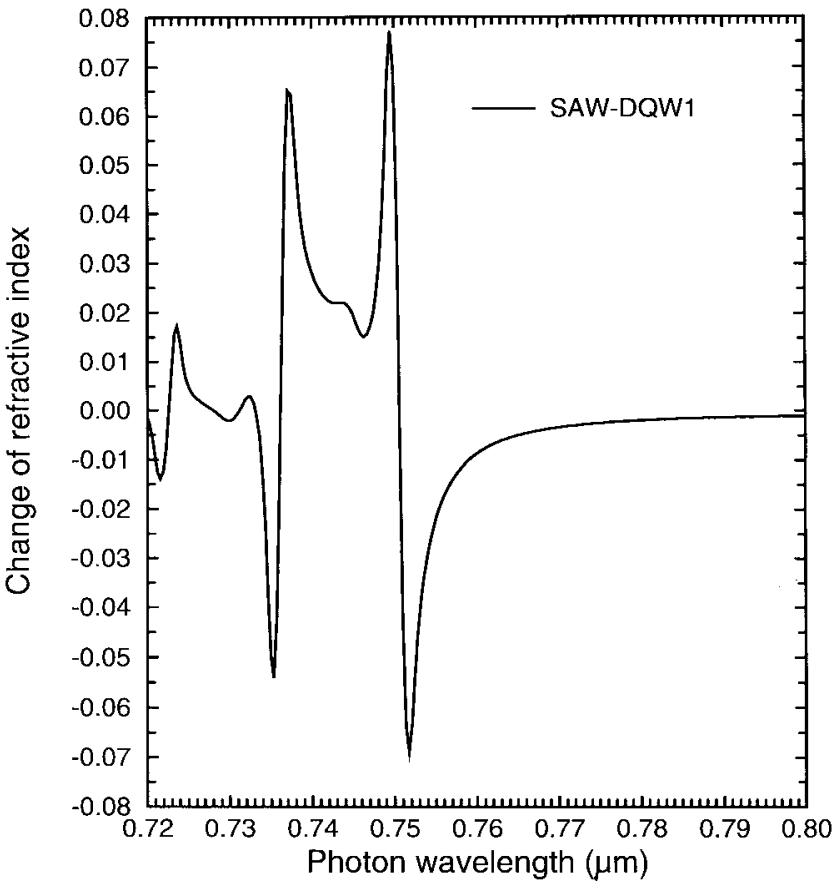

Fig. 7. The change of refractive index $\Delta n$ spectra of the first DQW's in the active region of the SAW phase modulator.

is more dominant, as compared to the intensity modulation. However, it is important to note that both $\Delta n_{\mathrm{eff}}$ and $\Delta \phi$ reduce when $\lambda_{\text {op }}$ increases and thus weakens the phase modulation. $\lambda_{\mathrm{op}}$ is finally selected at $0.76 \mu \mathrm{m}$ so that $\Delta n_{\mathrm{eff}}$ and $\Delta \phi$ values of $0.521 \times 10^{-2}$ and $2.15 \mathrm{rad}$, respectively, are obtained when $\beta_{\text {mod }}$ is up to 12.2 .
TABLE II

Modulation Properties of the Five-DQW Phase Modulator. SAW Aperture is $50 \mu \mathrm{m}$

\begin{tabular}{llllllll}
\hline$\lambda_{\text {op }}(\mu \mathrm{m})$ & $\begin{array}{l}\alpha_{\text {loss }} \\
\left(\mathrm{cm}^{-1}\right)\end{array}$ & $\begin{array}{l}\Delta \alpha_{\text {eff }} \\
\left(\mathrm{cm}^{-1}\right)\end{array}$ & $\begin{array}{l}\mathrm{n}_{\text {eff }} \\
(\mathrm{SAW})\end{array}$ & $\begin{array}{l}\mathrm{n}_{\text {eff }}(\text { no } \\
\mathrm{SAW})\end{array}$ & $\begin{array}{l}\Delta \mathrm{n}_{\text {eff }} \\
\left(10^{-2}\right)\end{array}$ & $\begin{array}{l}\Delta \phi \\
(\mathrm{rad})\end{array}$ & $\beta_{\text {mod }}$ \\
\hline 0.755 & 365 & 329 & 3.12298 & 3.10914 & 1.380 & 5.76 & 6.9 \\
0.76 & 136 & 70 & 3.12685 & 3.12163 & 0.521 & 2.15 & 12.2 \\
0.765 & 107 & 26 & 3.11325 & 3.11033 & 0.292 & 1.20 & 17.9 \\
0.77 & 71 & 13 & 3.10325 & 3.10134 & 0.192 & 0.78 & 23.4 \\
0.775 & 52 & 7.8 & 3.09524 & 3.09385 & 0.140 & 0.57 & 29.1 \\
0.78 & 41 & 5 & 3.08807 & 3.08699 & 0.108 & 0.43 & 34.7 \\
\hline
\end{tabular}

To compare these results with a SAW phase modulator using a five-period single QW as the active region [3], the optimized DQW has 10 times larger $\Delta n_{\text {eff }}$. In addition, since only a small change of the SAW induced potential (equivalent to a linear applied electric field of $\sim 20 \mathrm{kV} / \mathrm{cm}$ ) is required, the SAW with a long $\lambda_{\text {SAW }}$ of $15 \mu \mathrm{m}$ and a low SAW power of $3 \mathrm{~mW}$ can be used. Longer $\lambda_{\mathrm{SAW}}$ and lower SAW power simplifies the fabrication of the SAW transducer. Consequently, the asymmetric DQW phase modulator using SAW is an acoustooptical device with a large phase modulation.

\section{CONCLUSIONS}

In this paper, an optimized DQW optical phase modulator using SAW has been investigated theoretically. The optimized asymmetric DQW structure, i.e., Z30/15/60, X0.0/1.0/0.18 and isolation layers with $0.52 \mathrm{Al}$ fraction, is proposed as the fiveperiod DQW active region of the phase modulator for a SAW with $\lambda_{\mathrm{SAW}}$ and power of $15 \mu \mathrm{m}$ and $3 \mathrm{~mW}$, respectively. The results show that the large change of the SAW induced potential at the top surface can be utilized. Meanwhile, the five-DQW subband structure is tilted uniformly so that the refractive index change produced by each DQW is almost identical. In addition, the change of the effective refractive index for the optimized DQW is $\sim 10^{-2}$ which is 10 times that of a structure containing five single QW's of $\sim 10^{-3}$ [3] and 100 times that of a conventional AlGaAs bulk SAW modulator [22], i.e., a larger phase modulation is obtained in the DQW structure. Consequently, the asymmetric DQW phase modulator using SAW becomes an attractive candidate in the development of QW active optoelectronic devices and circuits.

It is important to note that, since the DQW structure is optimized by using an uniform applied field, the analysis in the optimization can also serve as a guide for developing an electrical biased DQW phase modulator, and the optimized DQW structure is also applicable for the use of a low-bias DQW phase modulator.

\section{ACKNOWLEDGMENT}

The authors would like to acknowledge the technical support of the Center for Vision, Speech, and Signal Processing (CVSSP) of the University of Surrey, U.K.

\section{REFERENCES}

[1] A. Van Der Lugt, Ed., "Special section on acousto-signal processing," Proc. IEEE, vol. 69, pp. 48-118, 1981. 
[2] T. Gryba and J. E. Lefebvre, "Optimization of MQW structures for acousto-optic absorption modulators," Proc. Inst. Elect. Eng., vol. 141, pt. J, pp. 62-64, 1994.

[3] W. C. H. Choy, E. H. Li, and B. L. Weiss, "AlGaAs/GaAs quantum well electro-optic and electro-absorptive modulators using surface acoustic wave," J. Appl. Phys., vol. 83, pp. 858-866, 1998.

[4] H. Feng, M. Sugiyama, J. P. Peng, K. Tada, and Y. Nakano, "Strong exciton absorption peak enhancement without red shift of absorption edge in $\mathrm{Al}_{0.3} \mathrm{Ga}_{0.7} \mathrm{As} / \mathrm{GaAs}$ five-step asymmetric coupled quantum well with modified potential," Jpn. J. Appl. Phys., vol. 36, pp. L855-L856, 1997.

[5] N. Susa, "Electric-field-induced refractive index changes in threestep asymmetirc coupled quantum wells," J. Appl. Phys., vol. 73, pp. 8463-8470, 1993

[6] Y. Kim, W. D. Hunt, F. S. Hickernell, R. J. Higgins, and C. K. Jen, "ZnO films on 001-Cut $\langle 110\rangle$-propagating GaAs substrates for surface acoustic wave device applications," IEEE Trans. Ultrason., Ferroelect., Fre. Contr., vol. 42, pp. 351-361, 1995

[7] Y. Kim and W. D. Hunt, "Acoustic fields and velocities for surfaceacoustic-wave propagation in multi-layered structures: An extension of the Laguerre polynomial approach," J. Appl. Phys., vol. 68, pp. 4993-4997, 1990.

[8] W. C. H. Choy and B. L. Weiss, "Interdiffusion induced modification of surface-acoustic-wave AlGaAs/GaAs quantum-well modulators," IEEE Select. Topic J. Quantum Electron., accepted for publication.

[9] V. M. Ascher, R. M. M. Matheig, and R. D. Russell, Numerical Solutions of Boundary Value Problems for Ordinary Differential Equations. Englewood Cliffs, NJ: Prentice-Hall, 1988, pp. 478.

[10] W. H. Press, S. A. Teukolshy, W. T. Vetterling, and B. P. Flannergy, Numerical Recipes in $C$, the Art of Scientific Computing, 2nd ed Cambridge, U.K.: Cambridge Univ., 1994.

[11] A. R. Adams and D. J. Dunsan, "Analysis and design of low-dimensional structures and devices using strain: I. Hydrostatic pressure effects," Semicond. Sci. Technol., vol. 5, pp. 1194-1201, 1990.

[12] T. Kamizato and M. Matsuura, "Excitons in double quantum wells," Phys. Rev. B, vol. 40, pp. 8378-8384, 1989.

[13] I. Galbraith and G. Duggan, "Exciton binding energy and external-fieldinduced blue shift in double quantum wells," Phys. Rev. B, vol. 40, pp. 5515-5521, 1989.

[14] W. C. H. Choy and E. H. Li, "Polarization insensitive optical modulation in a diffused parabolic-like quantum well," Appl. Opt., vol. 37, pp. 1674-1681, 1998
[15] W. C. H. Choy, B. L. Weiss, and E. H. Li, "The AlGaAs/GaAs quantum well electrooptic phase modulator with disorder delineated optical confinement," IEEE J. Quantum Electron., vol. 34, pp. 84-92, 1998.

[16] W. C. H. Choy and E. H. Li, "The applications of interdiffused quantum well in normally-on elecro-absorptive Fabry-Perot reflection modulation," IEEE J. Quantum Electron., vol. 33, pp. 382-393, 1997.

[17] W. C. H. Choy, E. H. Li, and B. L. Weiss, "Optical properties of $\mathrm{AlGaAs} / \mathrm{GaAs}$ and InGaAs/InP double quantum wells," unpublished.

[18] S. Adachi, "Optical-properties of $\mathrm{Al}_{x} \mathrm{Ga}_{1-x}$ As alloys," Phys. Rev. B vol. 38, pp. 12345-12352, 1988.

[19] G. J. Sonek, J. M. Ballantyne, Y. J. Chen, G. M. Carter, S. W. Brown, E. S. Koteles, and J. P. Slaerno, "Dielectric properties of GaAs/AlGaAs multiple quantum well waveguides," IEEE J. Quantum Electron., vol. QE-22, pp. 1015-1018, 1986

[20] T. Hausken, R. H. Yan, R. I. Simes, and L. A. Coldren, "Relating the chirp parameter to the number of quantum wells in GaAs/AlGaAs waveguide modulators," Appl. Phys. Lett., vol. 55, pp. 718-720, 1989.

[21] D. Atkinson, G. Parry, and E. J. Austin, "Modeling of electroabsorption in coupled quantum wells with applications to low-voltage optical modulation," Semicond. Sci. Technol., vol. 5, pp. 516-524, 1990.

[22] R. G. Hunsperger, Integrated Optics, Theory and Technology. New York: Springer-Verlag, 1985, ch. 9.

Wallace C. H. Choy, for biography, see p. 92 of the January 1998 issue of this JOURNAL.

E. Herbert Li (S'87-M'88-SM'95), for photograph and biography, see p. 990 of the June 1998 issue of this JourNaL.

Bernard L. Weiss (S'72-M'74-SM'94), for biography, see p. 92 of the January 1998 issue of this JOURNAL. 